\title{
Wortart und Kongruenz
}

Bis auf einige allgemeine und sehr vorsichtige Folgerungen wissen wir bekanntlich wenig über die Syntax der uralischen Grundsprache. Eine dieser Schlussfolgerungen betrifft die Kongruenz zwischen Attribut und Bezugswort. Nach dem Zeugnis unserer verwandten Sprachen war die Kongruenz unbekannt; wo wir sie heute dennoch antreffen, kann sie in jedem Fall als Ergebnis einer sekundären Entwicklung angesehen werden. (Vgl. Hajdú, Bevezetés az uráli nyelvtudományba [S. 88]. Budapest 1966.)

Innerhalb der heutigen finnisch-ugrischen Sprachen ist es beispielsweise nicht üblich, von einer Kongruenz in der attributiven Konstruktion des Ungarischen zu sprechen, während dagegen im Finnischen eine vollständige Kongruenz existiert.

Im Zusammenhang mit den Wortarten werden in den ungarischen Grammatiken die substantivischen und adjektivischen Kategorien allgemein mit ziemlicher Sicherheit behandelt. Hier handelt es sich um ein Substantiv, dort um ein Adjektiv. Nun bilden aber Adjektiv und Substantiv nicht in jeder Beziehung einander strikt gegenüberstehende und separierte Kategorien. (Ich möchte hier jetzt nicht in Einzelheiten gehen, zumal ich das in anderem Zusammenhang bereits getan habe. S. Magyar Nyelvészek Konferenciája. Nyíregyháza 1977.)

Die Differenzierung der Wortarten und damit auch ihre Differenzierbarkeit ist eine höchst komplizierte Frage: sie setzt sich aus einer ganzen Reihe von strukturellen, semantischen u.a. Teilmomenten zusammen. Diese Feststellung gilt ganz besonders im Falle des Substantivs und des Adjektivs. Die attributive Konstruktion baut sich aber gerade aus ihnen auf. Deshalb ist es so wichtig, wie sich diese Worttypen verhalten, 
wenn sie in einer syntaktischen Konstruktion miteinander verbunden sind. D.h.: was bedeutet die Kongruenz vom Standpunkt der Wortarten aus gesehen?

Die Auffassung der ungarischen Grammatiken lässt sich folgendermassen zusammenfassen: in den syntaktischen Konstruktionen des attributiven Typs wird das Adjektiv (denn diese Wortart zeigt die grössere Neigung, eine attributive Funktion auszufüllen) nicht flektiert. Flexionsendungen erhält nur das Bezugswort (und das ist i.a. ein Substantiv).

Z.B.: öreg postást 'den alten Briefträger'

öreg postások 'die alten Briefträger' (Nom.)

öreg postásokat 'die alten Briefträger' (Akk.)

Es gibt also keine Kongruenz! Von dieser Regel gibt es jedoch eine Ausnahme, die hinsichtlich ihrer Anwendungsfrequenz nicht zu allgemein ist: die Apposition. In dem Fall folgt das Attribut nach dem Bezugswort.

Z.B.: postást, öreget 'einen Briefträger, einen alten' postások, öregek 'Briefträger, alte'

Es schadet nicht, hier auch Formen mit dem bestimmten Artikel anzuführen.

\section{Z.B.: a postást, az öreget 'den Briefträger, den alten' a postásokat, az öregeket 'die Briefträger, die alten'}

Mit diesem Beispiel gelingt es wohl, die wortartmässige Zugehörigkeit des als Apposition stehenden Wortes zu klären. Wie aber gelangt ein Artikel vor ein Adjektiv, wo doch die Aufnahme des Artikels als substantivische Eigenschaft gilt?

Wenn wir zu unseren obigen Beispielen zurückkehren, können wir feststellen, dass in der Konstruktion öreg postások alles klar ist. Hier ist öreg das Adjektiv, postások das Substantiv. Wenigstens sieht es so aus. Denn aus denselben Wörtern kann auch eine andere Konstruktion entstehen, z.B. a postás öregek 'die Briefträger-Alten'. D.h. ein und dasselbe Wort kann in beiden Funktionen vorkommen. Ist nun das postás in unserem letzten Beispiel ein Substantiv oder ein Adjektiv? Vorläufig lassen wir auch diese Frage offen. 
Vor der Beantwortung der aufgekommenen Fragen müssen wir uns noch ein wenig anderswo umsehen. Wie oben bereits angedeutet wurde, werden die finnischen Attribute abweichend vom Ungarischen flektiert. Bis auf einige wenige Ausnahmen funktioniert die Kongruenz vollständig. Unter den Ausnahmen denke ich an solche Fälle, wo das als Attribut stehende Wort überhaupt nicht flektiert wird (z.B. pikku 'klein, winzig') oder wo das Attribut und das Bezugswort eine eigentümliche Disharmonie aufweisen (z.B. samalla tavoin 'auf dieselbe Weise'). Das letzte Beispiel kann aber auch als spezifische Manifestation der Kongruenz aufgefasst werden. Fred Karlsson verwendet in seiner Finsk grammatik (Suomalaisen Kirjallisuuden Seura, Helsinki 1978) bei der Betrachtung der Konstruktion der Wörter nur die Kategorie des Nomens. (Darauf baut sich beispielsweise ein ganzes Kapitel: "Böjningstyper för nomen", Flexionstypen für Nomina.) Über die Kongruenz schreibt er folgendes:

'Unter dem Attribut werden Bestimmungen des Substantivs verstanden. Es gibt zwei Hauptarten des Attributs, nämlich das Pronominalattribut und das Adjektivattribut. Alle diese Attribute müssen in Numerus und Kasus mit ihrem Bezugssubstantiv kongruieren. Das Attribut steht vor seinem Bezugswort." (Übersetzt aus dem schwedischen Original.)

\section{Z.B.: sininen kukka 'blaue Blume' siniset kukat 'blaue Blumen'}

Die in den finnischen attributiven Konstruktionen beteiligten Adjektive und Substantive können nicht flexibel ihre Funktionen wechseln. Ein und dasselbe Wortelement ist wesentlich weniger frei als im Ungarischen. Die syntaktisch redundante Kongruenz schlägt die Wörter in Fesseln, mitunter bis an die Grenze des Absurden (z.B. yhdet sakset 'eine Schere').

Die ungarische Sprache zeugt davon, dass man die unvollständige Differenzierung der Wortarten auch beim heutigen Stand der Sprache nicht ausser acht lassen kann. Darauf weist eigentlich auch unser Beispiel vom öreg postás und postás öreg hin, wo letzten Endes das öreg entsprechend den Funktionen einmal als Adjektiv, ein andermal als Substantiv auftreten kann.

Die Kongruenz geht denn auch wahrscheinlich auf das Konto 
dieser Undifferenziertheit. Der ursprünglich nur an dem Bezugswort vorkommende Zusatz konnte nur dann auch auf die anderen Wörter in dieser Konstruktion übergehen, wenn sie den wortartmässigen Grundvoraussetzungen entsprachen. Die morphematischen Mittel also trugen nur zu einer ähnlichen Bildung der ursprünglich unvollkommen differenzierten Wortarten bei. Die spezifische Rückwirkung davon war, dass sich die Wortarten stärker als erwartet gegeneinander abgrenzten und erstarrten.

Oder eigentlich standen im Falle der attributiven Kongruenz die zwei Funktionen am Anfang, die des Attributs und die des Bezugswortes. In diese Funktionen konnten sich die Nomina frei einfügen, ohne dass sie ein Adjektiv oder Substantiv sein mussten. Die Adjektivität und die Substantivität begann nur als zweitrangiges Element zu erscheinen. Das Auftreten in der attributiven Funktion gelangte im Laufe der Zeit zu den distinktiven Merkmalen des Adjektivs. Beziehungsweise: was die attributive Rolle ausfüllte, wurde zum Adjektiv und umgekehrt. Die Wortgestalt selbst aber war auch weiterhin zweitrangig: sie konnte flexibel von einer Wortart in die andere wechseln. Von hier stammt die Merkwürdigkeit der Beispiele des öreg postást und des postás öreget.

Es entwickelten sich also die Wortartkategorien, doch bedeutete das auch weiterhin nicht die Geschlossenheit der Einzelwörter. Und diese Tatsache trug wahrscheinlich bei zur Aufrechterhaltung der Kongruenzlosigkeit.

Im Finnischen nahm die Entwicklung eine etwas andere Richtung. Die attributive Konstruktion wurde durch die Kongruenz auch formal einheitlich.

Im Zusammenhang mit der Apposition ist noch einiges ungeklärt. Osmo Ikola unterscheidet im "Nykysuomen käsikirja" auch für das Finnische zwischen Attribut und Apposition. In dieser Konzeption ist "die Apposition ein Substantiv, das neben seinem Bezugswort unflektiert vorkommt oder mit ihm im gleichen Kasus und Numerus steht". In der ungarischen Apposition tritt ebenfalls das Substantiv auf und nicht das Adjektiv. Die potentielle Änderung der Wortart wurde oben bereits behandelt. Das als Apposition verwendete Wort wird in 
seiner Eigenschaft als Substantiv fähig, die für ein Substantiv typischen Endungen und Artikel aufzunehmen. Eine solche Konstruktion hat tatsächlich zwei Kerne (a postások, az öre$g e k$ ), was übrigens auch durch die verschiedenen prosodischen Elemente unterstützt wird. In Wirklichkeit ist das dann auch kein Attribut, sondern selbst ein Bezugswort. Die Appositionskonstruktion spricht also tatsächlich in nichts dagegen, dass das ungarische Adjektiv in der attributiven Konstruktion nicht kongruiert.

Zusammenfassend kann also gesagt werden, dass sich die adjektivische und substantivische Klasse im Ungarischen und Finnischen in vieler Hinsicht bemerkenswert anders verhält. Der Grad der Differenziertheit unterscheidet sich; dabei hat zweifellos auch die Kongruenz eine Rolle gespielt.

Warum verhalten sich nun die Possessivsuffixe in beiden Sprachen auf eine eigene Weise? Die finnische Possessivflexion gehört nicht in den Bereich der Kongruenz.

Z.B.: sininen kukkani 'meine blaue Blume'.

Im Ungarischen weist die Appositionskonstruktion ebenfalls überraschende Beispiele auf.

Z.B.: a postásom, az öreg 'mein Briefträger, der alte'.

Es erklärt sich wahrscheinlich syntaktisch, dass die Possessivendung nur am Bezugswort erscheint. Das besitzanzeigende Attribut ist syntaktisch und semantisch gesehen etwas anderes als das Eigenschafts-Attribut. Ausserdem müssen wir aber annehmen, dass die bislang skizzierte unvollständige Differenzierung der Wortarten keine vollkommene Vermischung bedeutete. Die distinktiven Merkmale der Wortarten mussten sich in dem Masse klären, dass sich die possessive Personalendung bei ihrem Erscheinen in die substantivischen Merkmale einreihte. Hinsichtlich ihres Alters ist demnach die Possessivendung relativ neu, jedenfalls kann sie erst auf die Zeit nach vollzogener Grunddistinktion zwischen Adjektiv und Substantiv datiert werden. Deshalb kann sie nicht in der Kongruenz vorkommen. Doch darüber ein andermal ausführlicher. 